\title{
Fast Initialization of Equalizers for VSB-Based DTV Transceivers in Multipath Channel
}

\author{
Jong-Moon Kim and Yong-Hwan Lee
}

\begin{abstract}
This paper proposes a fast initialization technique for equalization of 8-VSB-based digital television (DTV) signal in severe multipath channels. We consider the use of a modified decision feedback equalizer (MDFE) [1], [2] for fast initialization. The feedback filter (FBF) of the MDFE can be initialized simply by estimating the channel impulse response and only the feedforward filter (FFF) of the MDFE need training for initialization. To overcome the shortage of the training sequence in the VSB DTV signal, we propose a new initialization method by generating a virtual training signal to initialize the FFF of the MDFE. Simulation results show that the proposed scheme can fast initialize the equalizer using less than 5000 symbols, while providing the receiver performance comparable to that of conventional schemes.
\end{abstract}

Index Terms-Decision feedback equalizers, digital TV, multipath channels.

\section{INTRODUCTION}

$\mathbf{T}$ ERRESTRIAL broadcasting of digital television (DTV) signal experiences multipath channel with a delay of up to 18 microseconds corresponding to a span of 200 symbols or more [3]. It is well known that it takes a long time to train an equalizer under such a severe channel condition. Since the length of training signal in the vestigial sideband (VSB) DTV system is not sufficiently long, the use of blind equalization has widely been considered in VSB-based DTV receivers [3]. Blind equalizers do not require training sequence, but they may require a long time for initial startup. Moreover, they may not work properly in severe multipath channels with nonminimum phase.

To fast initialize the equalizer, we consider the use of a modified decision feedback equalizer (MDFE) [1], where the feedback filter (FBF) module is processed prior to the feedforward filter (FFF) module. Since the coefficient of the FBF of the MDFE can be initialized using an estimate of the channel impulse response, the MDFE only needs to train the FFF, significantly reducing the overall initialization time [1]. Since fractionally spaced (FS) equalizers can provide the performance robust to the timing phase error, we consider the use of an FS MDFE [2].

The use of minimum mean squared error (MMSE) method can be applied to the initialization of the FFF, but it may require a large implemental complexity as the tap size of the FFF increases. Moreover, it may not provide stable performance due to

Manuscript received May 13, 2004; revised October 28, 2004.

J.-M. Kim is with the Digital TV Laboratory, LG Electronics, Seoul 151-818, Korea (e-mail: jmoon13@lge.com).

Y.-H. Lee is with the School of Electrical Engineering and INMC, Seoul National University, Seoul 151-600, Korea (e-mail: ylee@ snu.ac.kr).

Digital Object Identifier 10.1109/TBC.2004.841751

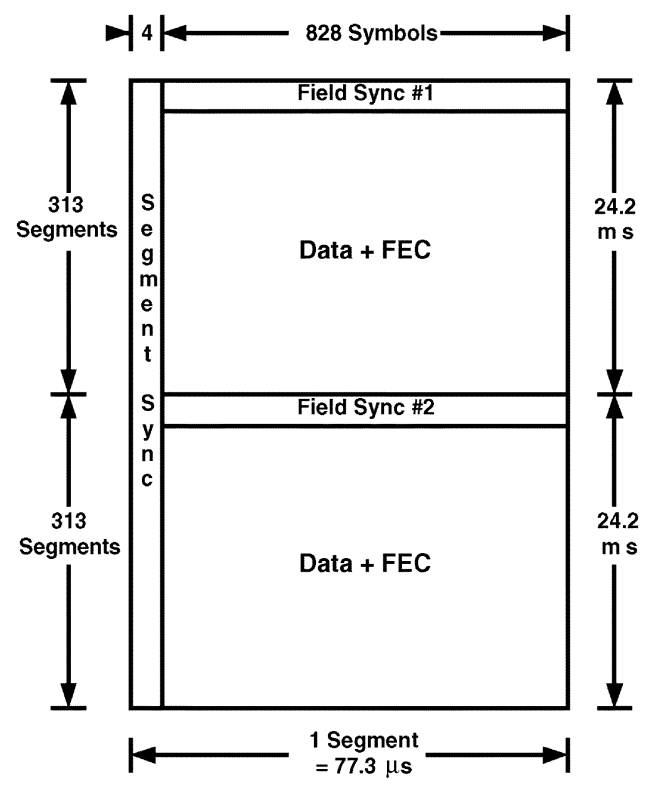

Fig. 1. Data frame of the VSB-based DTV signal.

a large eigenvalue spread in severe multipath channel [5]. To reduce the implementation complexity, the use of a partial MMSE (PMMSE) method was proposed in [2], where a few main coefficients of the FFF are first initialized before training all the coefficients. Since the FFF of the MDFE requires a large number of taps for reception of VSB-based DTV signal in severe multipath channel, the use of the PMMSE may not be appropriate. In this paper, we propose a new method for initialization of the MDFE. To overcome the shortage of the training sequence of the VSB-based DTV signal, we consider the use of a virtual training sequence additionally for the training of equalizer in a reference-directed mode. The virtual training sequence can be generated by passing a known sequence through the estimated channel response.

Following Introduction, Section II describes the system model. We propose a initialization scheme in Section III. The initialization performance of the proposed method is evaluated by computer simulation in Section IV. Finally, conclusions are summarized in Section V.

\section{SySTEM MODEL}

\section{A. DTV Frame Structure}

The frame structure of 8-VSB signal for DTV signal is illustrated in Fig. 1, [4]. The data frame comprises two data fields each of which contains 313 segments. The first segment of each data field is used as the field sync signal and the remaining segments are used for data signal. Each data segment comprises 
832 symbols, where the first four symbols are the segment sync symbol defined by $(5,-5,-5,5)$ and the remaining 828 symbols are trellis, RS-encoded and interleaved symbols drawn from eight-level pulse amplitude modulation (PAM) constellation $( \pm 1, \pm 3, \pm 5, \pm 7)$. The field sync segment is composed of four segment sync symbols, followed by a binary $( \pm 5) \mathrm{PN}$ sequence of period 511, three binary PN sequences of period 63 and other 128 binary symbols. The segment and field sync symbols are inserted in the data stream prior to pilot addition without channel coding.

\section{B. Modified Decision Feedback Equalizer}

We first consider the transmission of DTV signal over an additive Gaussian noise channel, whose input and output are represented as

$$
y(t)=\sum_{k} a_{k} h(t-k T)+v(t)
$$

where $T$ is the symbol time, $\left\{a_{k}\right\}$ denotes a real-valued data sequence, $h(t)$ is the overall impulse response of the channel including the transmit filter, physical channel and receive filter, and $v(t)$ denotes zero mean additive real Gaussian noise statistically independent of $\left\{a_{k}\right\}$.

Assume that the channel output $y(t)$ is over-sampled at a rate of $L / T$ and that the channel impulse response has a finite span over the time interval $\left[-K_{1} T, K_{2} T\right]$. Then, the input-output relationship for the discrete-time equivalent channel has a form of

$$
\mathbf{y}_{n}=\sum_{k=-K_{1}}^{K_{2}} a_{n-k} \mathbf{h}_{k}+\mathbf{v}_{n}
$$

where

$$
\begin{aligned}
& \mathbf{y}_{n} \triangleq[y((n+L-1 / L) T) \cdots y(n T)]^{\mathrm{T}}=\left[y_{n, L-1} \cdots y_{n, 0}\right]^{\mathrm{T}} \\
& \mathbf{h}_{n} \triangleq[h((n+L-1 / L) T) \cdots h(n T)]^{\mathrm{T}}=\left[h_{n, L-1} \cdots h_{n, 0}\right]^{\mathrm{T}} \\
& \mathbf{v}_{n} \triangleq[v((n+L-1 / L) T) \cdots v(n T)]^{\mathrm{T}}=\left[v_{n, L-1} \cdots v_{n, 0}\right]^{\mathrm{T}} .
\end{aligned}
$$

Here, the superscript $\mathrm{T}$ denotes the transpose of a matrix. For ease of analysis, we assume that the channel has a maximum amplitude at $h_{0,0}$. As a consequence, the channel impulse response (CIR) is not causal, but it can be made causal by introducing a delay of $K_{1} T$.

Assuming that the channel is time-invariant over a block interval of $F$ symbols, (2) can be rewritten in a matrix form, as seen in (4) at the bottom of the page, where the first and last subscript separated by a colon indicate the components of the vector.
In the FS-MDFE, the channel output $\mathbf{y}_{n+F-1: n}$ is fed to the feedback section to precancel the postcursor inter-symbol-interference (ISI)

$$
\begin{aligned}
\mathbf{x}_{n+F-1: n} & =\mathbf{y}_{n+F-1: n}-\mathbf{H}_{\text {post }} \hat{\mathbf{a}}_{n+\left(K_{1}+F-1\right)-\Delta-1: n-K_{2}} \\
& =\mathbf{y}_{n+F-1: n}-\mathbf{V}_{F-1: 0}(n)
\end{aligned}
$$

where $\mathbf{x}_{n+F-1: n}$ denotes the output of the FBF (i.e., the input of the FFF of the FS-MDFE), $\hat{a}_{n}$ is the detected data symbol, $\Delta$ indicates the decision delay of the FS-MDFE due to the channel and FFF, $\mathbf{H}_{\text {post }}$ is the FBF section represented as

$\mathbf{H}_{\text {post }} \triangleq\left[\begin{array}{cccccc}\hat{\mathbf{h}}_{\Delta-\left(K_{1}+F-1\right)+F} & \cdots & \hat{\mathbf{h}}_{K_{2}} & \mathbf{0} & \cdots & \mathbf{0} \\ \hat{\mathbf{h}}_{\Delta-\left(K_{1}+F-1\right)+F-1} & \cdots & & \hat{\mathbf{h}}_{K_{2}} & & \vdots \\ \vdots & & & & \ddots & \mathbf{0} \\ \hat{\mathbf{h}}_{\Delta-\left(K_{1}+F-1\right)+1} & & \cdots & \cdots & & \hat{\mathbf{h}}_{K_{2}}\end{array}\right]$

$\hat{\mathbf{h}}_{n}$ is the FBF tap coefficient obtained from the channel estimator, and $\mathbf{V}_{q}(n)$ is the postcursor ISI term related to the FFF tap coefficient

$$
\mathbf{V}_{q}(n) \triangleq \sum_{m=1}^{B} \hat{a}_{n+\left(K_{1}+F-1\right)-\Delta-m} \hat{\mathbf{h}}_{\Delta-\left(K_{1}+F-1\right)+m+q}
$$

Here, B equals to the tap size of the FBF divided by the oversampling ratio $L$.

Assuming perfect channel estimation and no decision error, (6) can be rewritten as

$$
\mathbf{x}_{n+F-1: n}=\mathbf{H}_{\text {pre }} a_{n+\left(K_{1}+F-1\right): n+\left(K_{1}+F-1\right)-\Delta}+\mathbf{v}_{n+F-1: n}
$$

where

$$
\begin{aligned}
& \mathbf{H}_{\text {pre }} \\
& \triangleq\left[\begin{array}{cccccc}
\mathbf{h}_{-K_{1}} & \mathbf{h}_{-K_{1}+1} & & \cdots & & \mathbf{h}_{\Delta-\left(K_{1}+F-1\right)+F-1} \\
\mathbf{0} & \mathbf{h}_{-K_{1}} & & \cdots & & \mathbf{h}_{\Delta-\left(K_{1}+F-1\right)+F-2} \\
\vdots & & \ddots & & & \vdots \\
\mathbf{0} & \ldots & \mathbf{0} & \mathbf{h}_{-K_{1}} & \ldots & \mathbf{h}_{\Delta-\left(K_{1}+F-1\right)}
\end{array}\right] .
\end{aligned}
$$

Define the FFF tap coefficient by

$$
\mathbf{w}^{\mathrm{T}} \triangleq\left[\mathbf{w}_{-(\mathbf{F - 1})}^{\mathrm{T}} \cdots \mathbf{w}_{-1}^{\mathrm{T}} \quad \mathbf{w}_{0}^{\mathrm{T}}\right]
$$

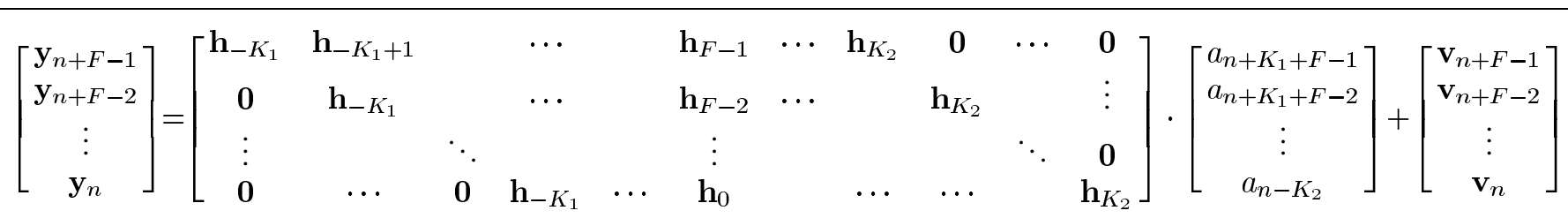

or

$$
\mathbf{y}_{n+F-1: n}=\mathbf{H}_{\left(K_{1}+K_{2}+F-1\right) \times(F-1)} \mathbf{a}_{n+K_{1}+F-1: n-K_{2}}+\mathbf{v}_{n+F-1: n}
$$




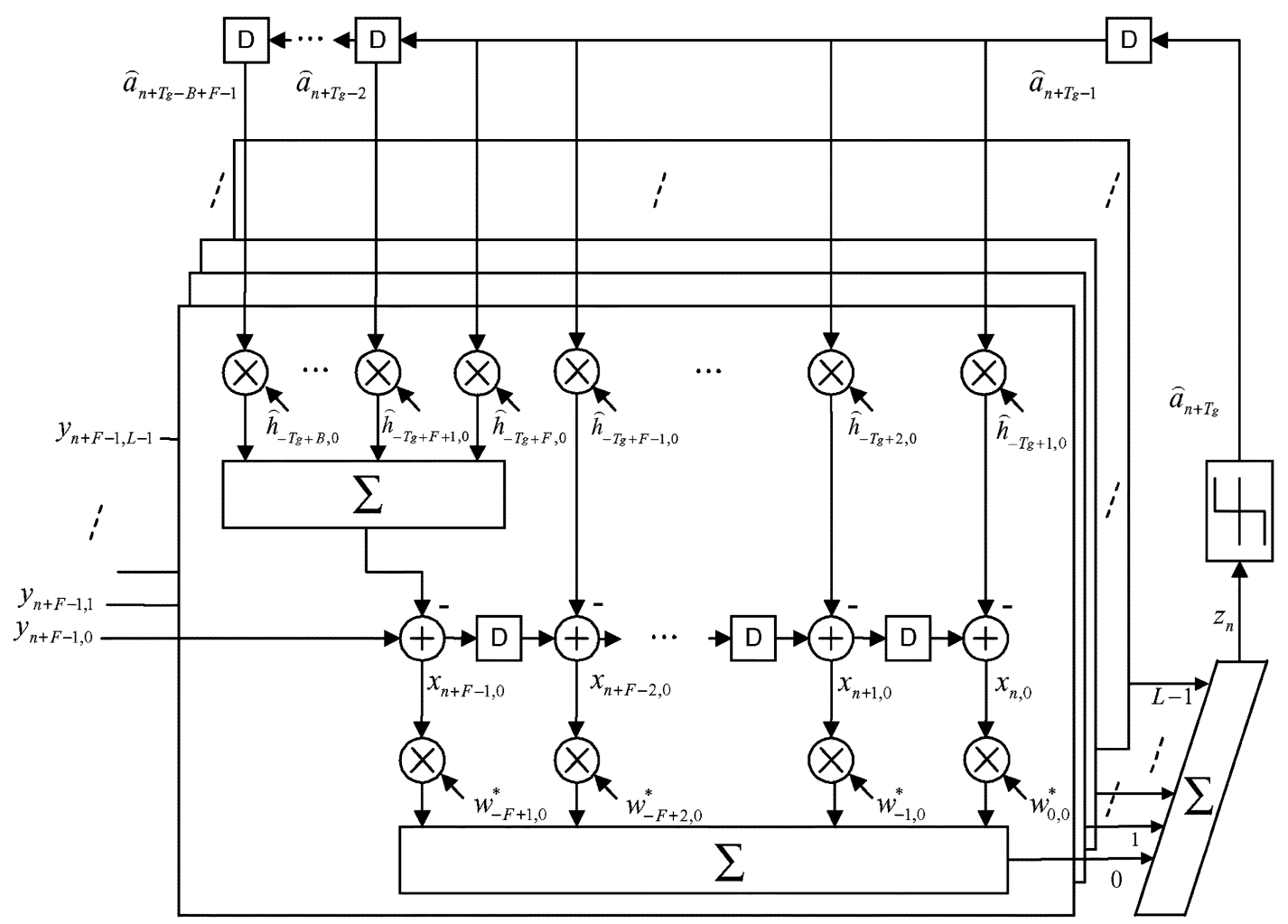

Fig. 2 Structure of fractionally spaced modified DFE.

where $\mathbf{w}_{-q}^{\mathrm{T}} \triangleq\left[w_{-q, L-1} \cdots w_{-q, 1} w_{-q, 0}\right]$. Then, the output of the FS-MDFE $z_{n}$ is represented as

$$
z_{n}=\mathbf{w}^{\mathrm{T}} \mathbf{x}_{n+F-1: n}
$$

and the corresponding error signal is

$$
\begin{aligned}
e_{n} & =a_{n+\left(K_{1}+F-1\right)-\Delta}-z_{n} \\
& =a_{n+\left(K_{1}+F-1\right)-\Delta}-\mathbf{w}^{\mathrm{T}} \mathbf{x}_{n+F-1: n} .
\end{aligned}
$$

For ease of description, define a new variable $T_{g}$ by

$$
T_{g} \triangleq\left(K_{1}+F-1\right)-\Delta
$$

that indicates the position of the main tap in the FFF. For example, $T_{g}=0$ implies that the position of the main tap is on $w_{0,0}^{*}$ of the FFF, $T_{g}=1$ implies $w_{-1,0}^{*}$, and so on. Fig. 2 depicts the structure of the FS-MDFE, where the FBF part is simplified by a recursive relation, as seen in (15) at the bottom of the page.

\section{INITIALIZATION OF THE MDFE}

The initialization of the MDFE requires the channel information. The CIR can be estimated using the field sync signal. The FBF of the MDFE can be initialized using an estimated CIR.

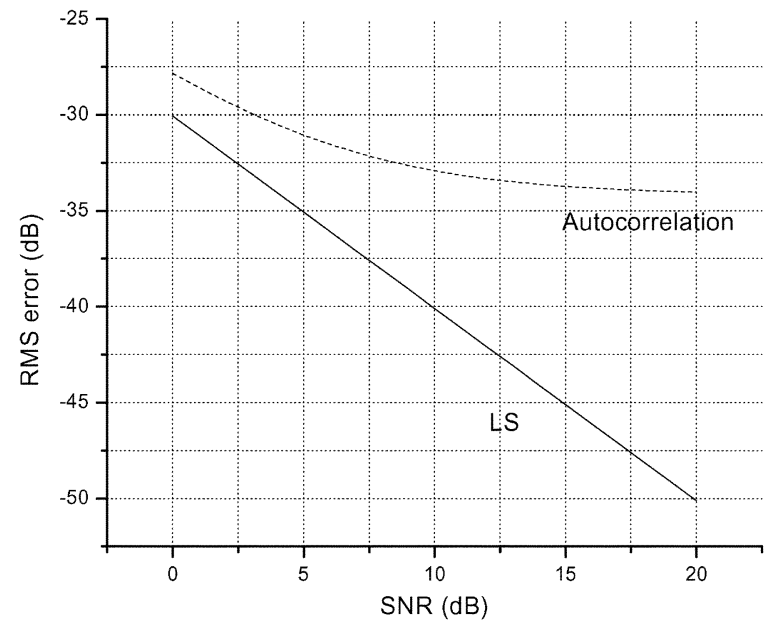

Fig. 3. RMS channel estimation error.

The FFF of the MDFE can be initialized by a training process or inverse operation of the channel correlation matrix [2].

The CIR can be estimated by two methods using the field sync signal. One is the use of autocorrelation method and the other is the use of least square (LS) algorithm. Since the PN sequence of length 511 is not periodic, the autocorrelation method may

$$
\mathbf{V}_{q-1}(n)=\left\{\begin{array}{ll}
\mathbf{V}_{q}(n-1)+a_{n+\left(K_{1}+F-1\right)-\Delta-1} \mathbf{h}_{q+\Delta-\left(K_{1}+F-1\right)}, & q=1,2, \cdots, F-1 \\
\sum_{m=1}^{B} a_{n+\left(K_{1}+F-1\right)-\Delta-m} \mathbf{h}_{m+\Delta-\left(K_{1}+F-1\right)+F-1}, & q=F
\end{array} .\right.
$$




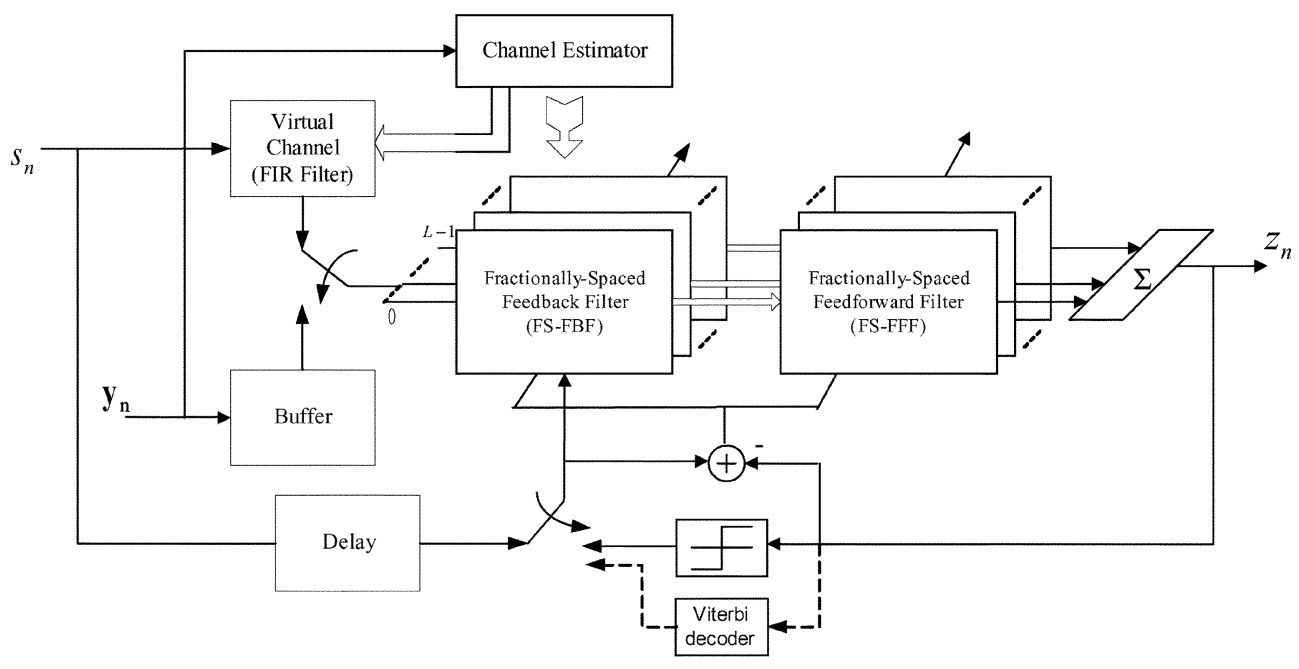

Fig. 4. Structure of the proposed initialization scheme.

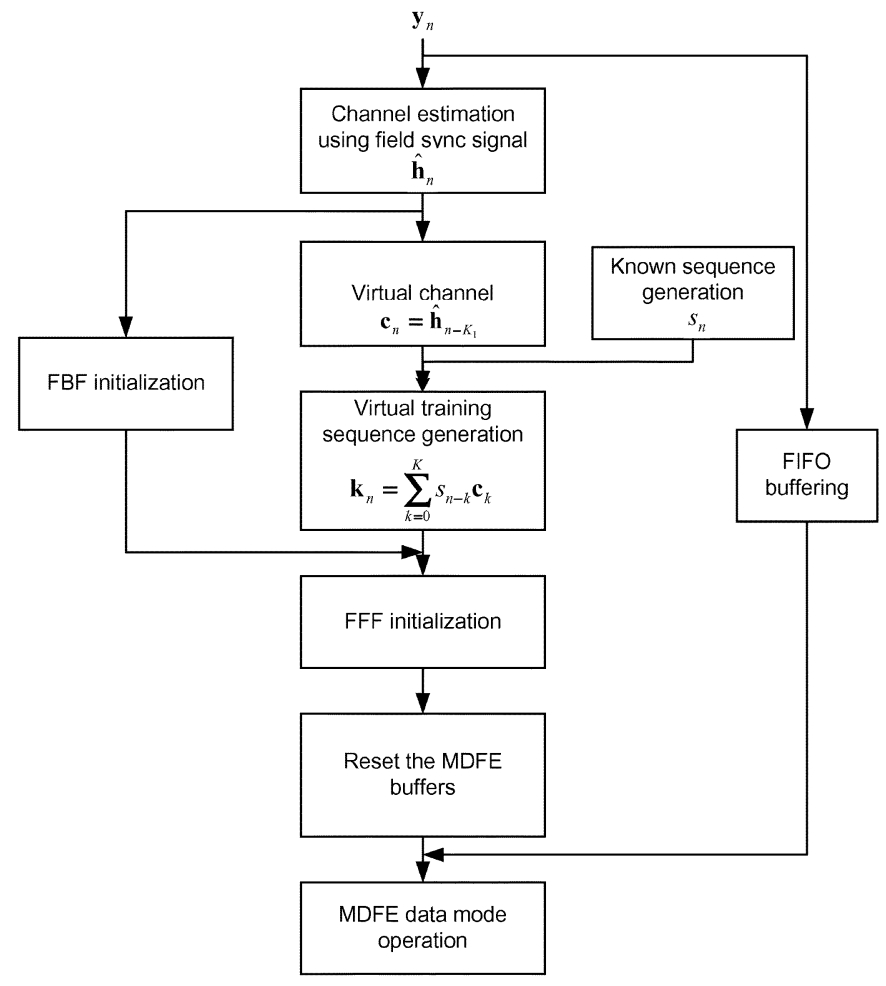

Fig. 5. Flow chart of the proposed initialization scheme.

not provide an accurate estimate. The LS estimate can easily be obtained by, for $N \geq K$

$$
\left[\begin{array}{c}
\hat{\mathrm{h}}_{-\mathrm{K}_{1}}^{\mathrm{T}} \\
\vdots \\
\hat{\mathrm{h}}_{0}^{\mathrm{T}} \\
\vdots \\
\hat{\mathrm{h}}_{\mathrm{K}_{2}}^{\mathrm{T}}
\end{array}\right]=\left[\begin{array}{c}
\mathrm{p}_{\mathrm{K}+\mathrm{N}-2} \cdots \mathrm{p}_{\mathrm{N}} \mathrm{p}_{\mathrm{N}-1} \\
\vdots \\
\mathrm{p}_{\mathrm{K}+\mathrm{n}-1} \cdots \\
\vdots \\
\cdots \\
\mathrm{p}_{\mathrm{K}-1} \cdots \mathrm{p}_{\mathrm{n}+1} \mathrm{p}_{\mathrm{n}} \\
\mathrm{p}_{1} \mathrm{p}_{0}
\end{array}\right]^{-1}\left[\begin{array}{c}
\mathrm{y}_{\mathrm{N}-1}^{\mathrm{T}} \\
\vdots \\
\mathrm{y}_{\mathrm{n}}^{\mathrm{T}} \\
\vdots \\
\mathrm{y}_{0}^{\mathrm{T}}
\end{array}\right]
$$

where $K\left(=K_{1}+K_{2}+1\right)$ denotes the CIR length and $\left\{p_{n}\right\}$ represents the field sync sequence. Note that the matrix inversion can be replaced by pseudo inversion when $N>K$, which can be precalculated [6]. Fig. 3 compares the channel estimation
TABLE I

SIMULATION PARAMETERS

\begin{tabular}{c|c}
\hline Over-sampling ratio $(L)$ & 2 \\
\hline Number of FFF coefficients $(F \times L)$ & 128 \\
\hline Number of FBF coefficients $(B \times L)$ & 300 \\
\hline Timing gear $\left(T_{g}\right)$ & 3 \\
\hline Least-Square channel estimation & $\begin{array}{c}N=550 \\
K=150\end{array}$ \\
\hline Virtual training duration & 5000 (symbol) \\
\hline $\begin{array}{c}\text { LMS step size of FFF for the } \\
\text { first 3000 symbols }\end{array}$ & 0.0007 \\
\hline $\begin{array}{c}\text { LMS step size of FFF for the } \\
\text { next 1000 symbols }\end{array}$ & 0.0003 \\
\hline $\begin{array}{c}\text { LMS step size of FFF for the } \\
\text { last 1000 symbols }\end{array}$ & 0.0001 \\
\hline LMS step size of FBF & 0.000005 \\
\hline
\end{tabular}

error of the two methods in terms of the input SNR. It can be seen that the autocorrelation method doesn't work well even at high SNR, but the LS method works well as the SNR increases.

Since the use of matrix inversion involves computational complexity, we consider the use of LMS training for initialization of the FFF. The field sync signal can be used for the LMS training, but its length is too short to sufficiently initialize the FFF. To overcome this problem, we generate an additional training sequence, called virtual training sequence at the receiver.

Fig. 4 depicts the structure of the proposed initialization scheme and Fig. 5 summarizes the process of the proposed scheme. We generate an additional training signal (called virtual training sequence) $\mathrm{k}_{n}$ by passing a known sequence $s_{n}$ through a virtual channel whose impulse response is set by the CIR estimated using the field sync signal, i.e.,

$$
\mathbf{k}_{n}=\sum_{k=0}^{K} s_{n-k} \mathbf{c}_{k}
$$

where $\mathbf{c}_{k}$ is the estimated CIR. The training sequence can be generated using $\underline{\text { a }}$ binary random sequence with white spectrum. Since the channel estimation can be assumed small in normal operating condition, the generated virtual training sequence can 


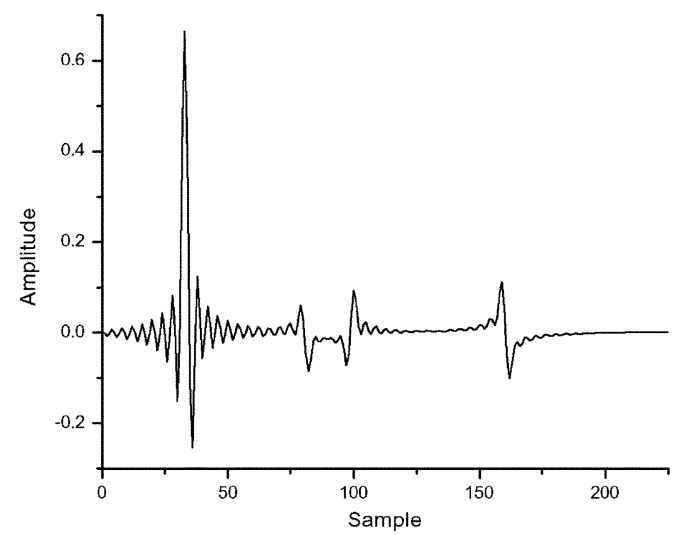

(a)

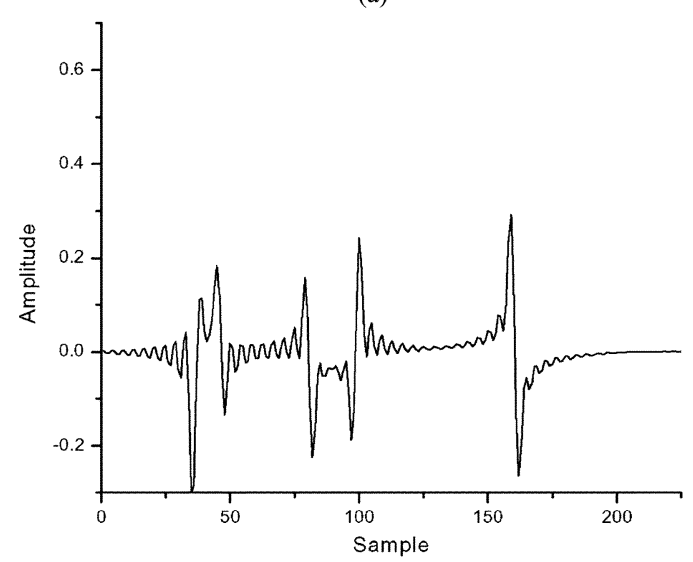

(b)

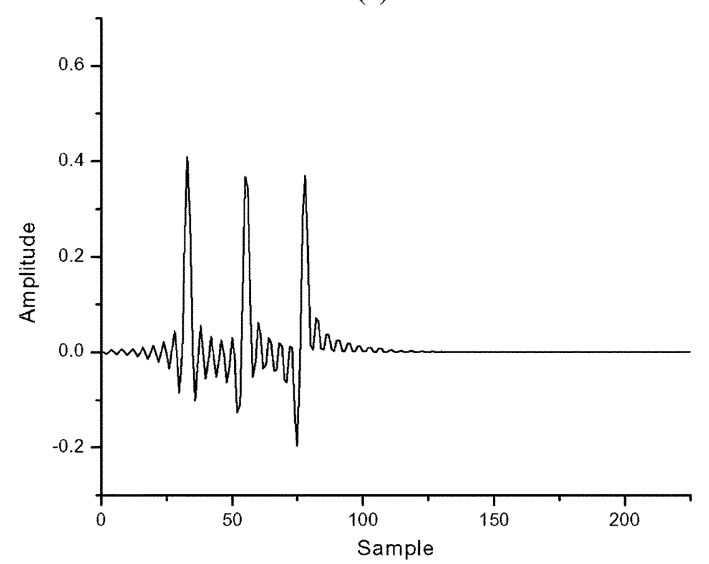

(c)

Fig. 6. Overall CIR of Brazil channels. (a) Brazil A channel, (b) Brazil D channel, (c) Brazil E channel.

be used as the training signal. Thus, the FFF can be trained using a conventional LMS method in a reference training mode. Since the channel condition is little changed during a short training interval, the proposed method can be applied to real situation.

Since the FFF is initialized under the channel condition at the time of channel estimation, it is necessary for the MDFE to start the data mode operation using data just after the channel estimation. It is also indispensable to maintain the continuity of the input signal for proper operation of the MDFE. Otherwise, the MDFE cannot properly operate due to disastrous error propagation. Thus, it is necessary to buffer (or store) the signal $y_{n}$ received while the FFF is trained using the virtual training signal.

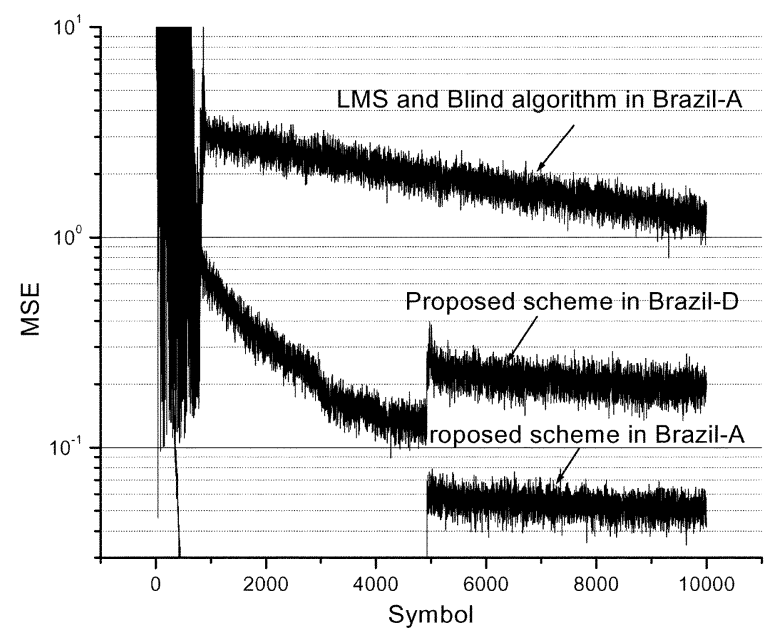

Fig. 7. Initialization performance of the proposed scheme.

After the FFF is initialized, it is necessary to reset the FFF and FBF buffers before the data mode operation. The MDFE generates the equalized output with a delay equal to the amount of the virtual training time, using the received signal buffered in a FIFO-type memory as shown in Fig. 4.

\section{Simulation Results}

The performance of the proposed initialization method is verified by computer simulation under the Brazil channel condition [7]. The simulation parameters for LS channel estimation and MDFE are summarized in Table I. The overall impulse response of typical Brazil channels, including the transmit filter, channel, and receiver filter, is depicted in Fig. 6.

Fig. 7 depicts the initialization performance of the MDFE in terms of the mean squared error (MSE), when the input SNR is $25 \mathrm{~dB}$. For comparison, we consider the use of a blind DFE with the same tap size as the MDFE. The blind DFE is first trained by an LMS algorithm using the field sync signal as the reference signal and then by a constant modulus algorithm (CMA) using the data signal. It can be seen that the proposed MDFE can be initialized within less than 5000 symbols even in severely ill condition like Brazil D-channel, but the blind equalizer requires more than 100000 symbols even in mild condition like Brazil-A channel. The use of the proposed MDFE requires an FIFO-type buffer of size 5000 symbols, corresponding to a processing delay of less than $0.5 \mathrm{~ms}$. After the initialization, it can be seen that the equalizer output is somewhat temporarily degraded. This is mainly due to the noise and convergence error by using a virtual channel instead of real channel.

Fig. 8 depicts the receiver performance in terms of the bit error rate (BER) under the simulation condition summarized in Table I. It can be seen that the receiver performance is significantly affected by the error propagation in the FBF. For reference, the BER with the use of decoder output without error as the input to the FBF (denoted as "correct FBF input") is also shown in Fig. 8. It can be seen that the use of the slicer output as the FBF input does not work well. To alleviate this problem, we consider the use of tentative decisions of the Viterbi decoder as the FBF input [8]. The tentative decision is obtained by slicing the present state signal with the minimum path metric in the Viterbi 


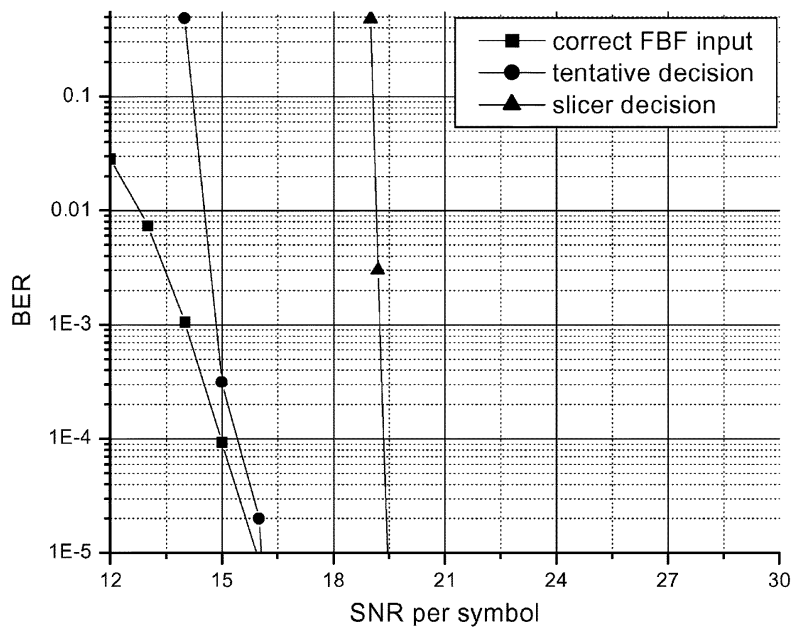

(a)

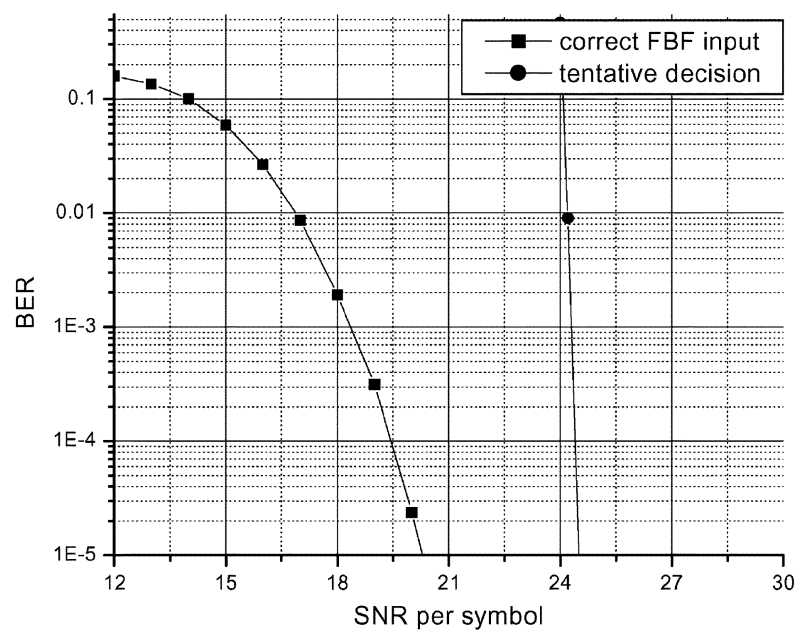

(b)

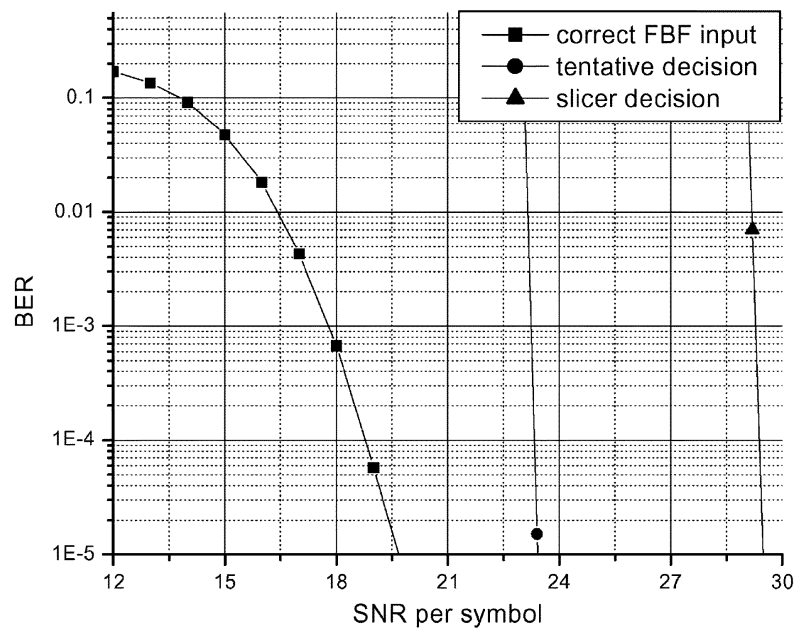

(c)

Fig. 8. BER performance with the use of MDFE (a) Brazil A channel, (b) Brazil D channel, (c) Brazil E channel.

decoder. It can be seen that the proposed MDFE can provide acceptable receiver performance at an SNR higher than $25 \mathrm{~dB}$ even in severe nonminimum phase multi-path channels.

The MDFE can provides good performance in Brazil A and $\mathrm{E}$ channels unless the tap size of the FFF is too small. However, the MDFE may need the use of an FFF with a large tap size in non-

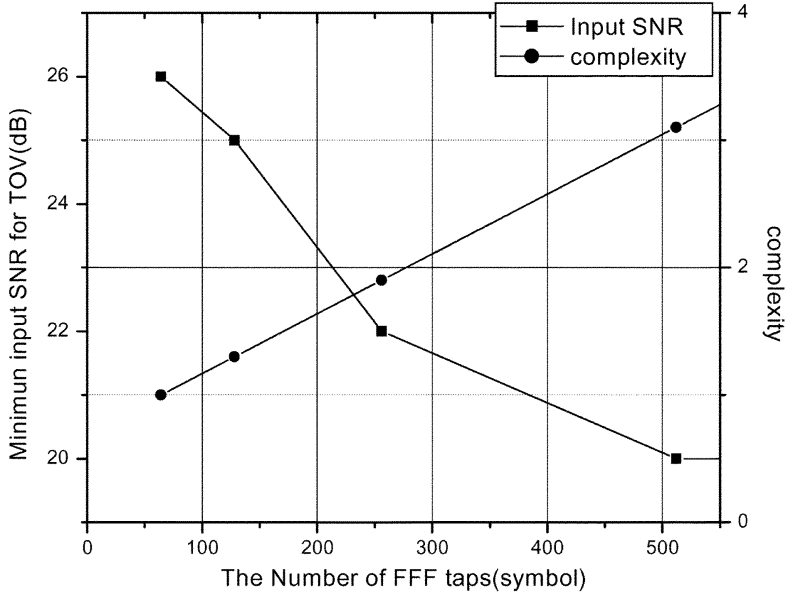

Fig. 9. Required minimum input SNR for the TOV.

minimum phase channel. Fig. 9 depicts the minimum input SNR for the threshold of visibility (TOV) in terms of the FFF tap size in Brazil D channel, where the complexity means the number of multiplications required for the MDFE. It can be seen that improved performance can be obtained in nonminimum phase channel by using an FFF with a significantly large tap size.

\section{CONCLUSION}

We have considered fast equalizer training for reception of VSB-based DTV signal. We have proposed the use of an MDFE that only needs to train the FFF. Since the length of the training sequence is too short, we have proposed the use of a virtual training sequence for training the MDFE. Simulation results show that the proposed MDFE can be fast trained within less than 5000 symbols time even in severe nonminimum phase channels.

\section{REFERENCES}

[1] S. Ariyavisitakul and L. J. Greenstein, "Reduced-complexity equalization techniques for broadband wireless channels," IEEE J. Select. Areas Commun., vol. 15, no. 1, pp. 5-15, Jan. 1997.

[2] H. Lee, S. Hong, Y. H. Lee, and G. B. Lee, "Fast training of fractionally-spaced modified decision feedback equalizer in slow frequency selective fading channels," in Proc. IEEE VTC Spring 2002, vol. 4, 2002 , pp. $1694-1698$

[3] M. Ghosh, "Blind decision feedback equalization for terrestrial television receivers," Proc. IEEE, vol. 86, no. 10, pp. 2070-2081, Oct. 1998

[4] ATSC Digital Television Standard, Doc. A/53B (2001, Aug.). [Online]. Available: http://www.atsc.org/stan\&rps.html

[5] S. Haykin, Adaptive Filter Theory, 4th ed, New Jersey: Prentice-Hall Inc., 2002.

[6] E. G. Goodaire, Linear Algebra, 1st ed: Prentice-Hall Inc., 2003.

[7] "Tests on Digital Television Systems: Third Report,", Mar. 25, 2000. SET/ABERT.

[8] S. Ariyavisitakul and Y. Li, "Joint coding and decision feedback equalization for broadband wireless channels," IEEE J. Select. Areas Commun., vol. 16, no. 9, pp. 1670-1678, Dec. 1998.

[9] N. Deshpande, "Fast recovery equalization techniques for DTV signals," IEEE Trans. Broadcast., vol. 43, no. 4, pp. 370-377, Dec. 1997.

[10] H. N. Kim, Y. T. Lee, and S. W. Kim, "Blind decision feedback equalization for VSB-based DTV receivers," IEEE Trans. Commun., vol. 48, no. 3, pp. 602-609, Aug. 2002.

[11] De Parthapratim, J. Bao, and T. Poon, "A calculation-efficient algorithm for decision feedback equalizers," IEEE Trans. Consumer Electron., vol. 45, no. 3, pp. 526-532, Aug. 1999.

[12] I. J. Fevrier, S. B. Gelfand, and M. P. Fitz, "Reduced complexity decision feedback equalization for multipath channels with large delay spreads," IEEE Trans. Commun., vol. 47, no. 6, pp. 927-937, Jun. 1999. 